\title{
Buried Superior Gluteal Artery Perforator Flap for Dead Space Coverage in the Sacral Area
}

\author{
Seung Heon Hong (ㅁ, Kyeong-Tae Lee (1) \\ Department of Plastic Surgery, Samsung Medical Center, Sungkyunkwan University School of Medicine, Seoul, Korea
}

\begin{abstract}
Partial sacrectomy is a common treatment for sacral sarcomas, which often results in a large defect and exposed rectal wall. The defect could be covered using a gluteus maximus (GM) advancement flap. However, seroma and wound dehiscence are often observed after GM advancement flap coverage, delaying the wound healing process. We present a case of buried superior gluteal artery perforator (SGAP) flap following GM muscle splitting. An 11-year-old male patient with epithelioid sarcoma in the sacrum underwent partial sacrectomy. The sacral defect size was $9 \times 6 \mathrm{~cm}$, and the GM muscle was intact. We designed a 7×4-cm elliptically-shaped SGAP flap skin paddle, after which perforator dissection was performed underneath the GM muscle. To minimize dead space, the GM muscle was split and the flap was de-epithelized and advanced to the posterior rectal wall. There were no wound complications during admission and the patient was discharged on postoperative day 16. No atrophy of the flap was found on postoperative magnetic resonance imaging, either. This case demonstrates that using a buried SGAP flap for covering dead space could be a good surgical method to cover wide sacral defects.
\end{abstract}

Keywords: Superior gluteal artery perforator flap; Sacrectomy reconstruction; Buried flap

\section{Introduction}

In the treatment of sarcoma in the sacral area, partial sacrectomy is often the procedure of choice in order to attain a wide safety margin while removing the tumors. After partial sacrectomy, usually the sacral defect is large and the rectal posterior wall is exposed. Properly protecting the rectal wall is important for the patient's quality of life. In partial sacrectomy, the skin is usually spared and the defect can be closed using a gluteus maximus (GM) advancement flap. Following reconstruction of a sacral defect by a GM advancement flap, however, there is 3.8\% incidence of seroma [1] and nearly $30 \%$ incidence of wound dehiscence [2], delaying wound healing and sometimes leading to wound infection. Meanwhile, full coverage with a vertical rectus abdominis flap is considered a good surgical option for covering the defect, but is known to have over $14 \%$ incidence of seroma and 35\% wound healing problems [3]. Here we present a case of buried superior gluteal artery perforator (SGAP) flap following GM muscle splitting to cover the dead space. Our investigation was conducted according to the principles expressed in the Declaration of Helsinki and informed consent was obtained from the patient and patient's guardian.

\section{Case}

An 11-year-old male patient had epithelioid sarcoma in the sacrum and underwent partial sacrectomy. The rectal posterior wall was exposed and the GM muscle was in-

\section{Case Report}

Received: October 29, 2019

Revised: November 10, 2019

Accepted: November 10, 2019

\section{Corresponding author:}

Kyeong-Tae Lee, M.D.

\section{Department of Plastic Surgery, Samsung Medical Center, Sungkyunkwan University School of Medicine, 81 Irwon-ro, Gangnam-gu, Seoul 06351, Korea \\ Tel: +82-2-3410-0220 \\ Fax: +82-2-3410-0036 \\ E-mail: skymaker26@gmail.com}

This is an Open Access article distributed under the terms of the Creative Commons Attribution Non-Commercial License (https://creativecommons.org/licenses/by-nc/4.0/) which permits unrestricted non-commercial use, distribution, and reproduction in any medium, provided the original work is properly cited.

(c) 2020 Korean Wound Management Society 
tact with a $9 \times 6-\mathrm{cm}$ sacral defect. Our team was contacted to perform coverage of the sacral defect. We designed a $7 \times 4-\mathrm{cm}$ elliptically-shaped SGAP flap skin paddle and performed flap perforator dissection underneath the GM muscle. To minimize dead space, the GM muscle around the perforator was split and the flap was passed through the split GM muscle, fully covering the posterior rectal wall. The flap was then de-epithelized and the split GM muscle was repaired (Figs. 1, 2). Both sides of the GM muscle were advanced to cover the deepithelized skin flap and the wound was closed.

There were no wound complications including seroma and wound dehiscence during admission, and the patient was discharged on postoperative day 16 . No post-discharge wound
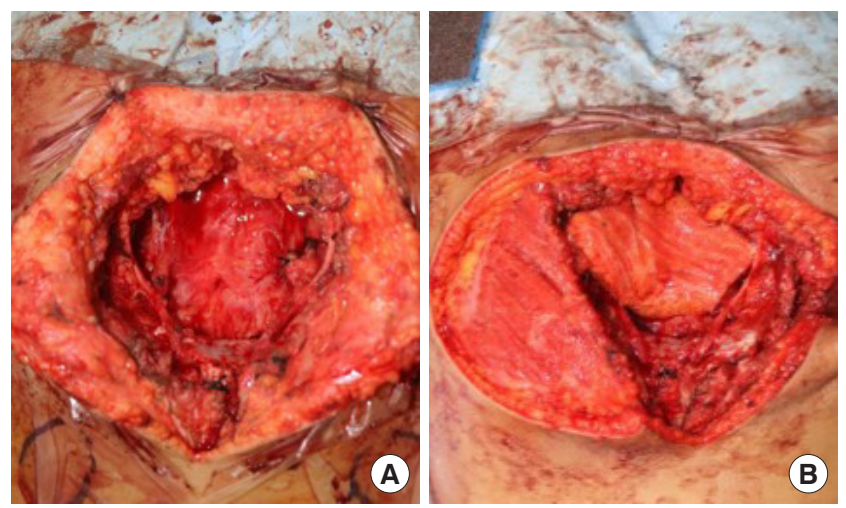

Fig. 1. Intraoperative photographs. (A) Sacral defect after partial sacrectomy. (B) Sacral defect covered by de-epithelization of superior gluteal artery perforator flap under the gluteus maximus muscle.

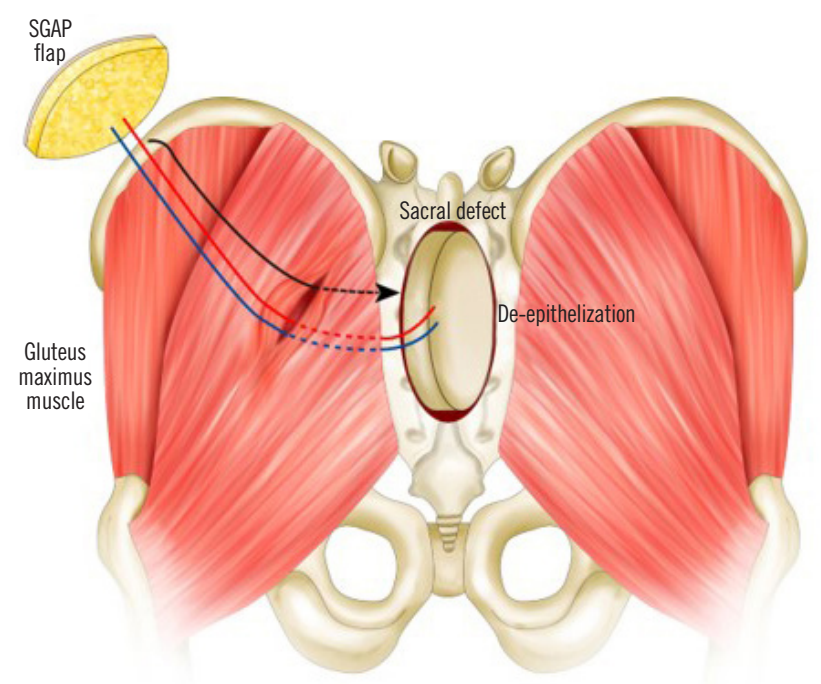

Fig. 2. Schematic image of buried perforator flap. Flap was deepithelized and tunneled through gluteus maximus muscle to cover the sacral defect. SGAP, superior gluteal artery perforator. complications were found (Fig. 3). Regular magnetic resonance imaging (MRI) was checked for 2 years postoperatively and no atrophy of the flap was found on imaging (Fig. 4).

\section{Discussion}

Sacral tumors are usually treated with partial sacrectomy. After sacrectomy, some tumors require radiation therapy, rendering the affected tissues more vulnerable to infection. Therefore, preventing possible risk factors of infection is especially important in reconstructing sacral defects. One of the postoperative infection risk factors is seroma, with an incidence rate as high as $3.8 \%$, occurring when there is dead space in the wound. For the typically sizeable post-sacrectomy defects, the dead space is difficult to cover with the popular reconstruction method using bilateral gluteal advancement flaps. This large dead space causes both functional and aesthetic problems; there is higher risk of seroma, and the contours of the buttock could be flattened in the sacral region, which could cause great stress for a young patient. Furthermore, reinforcing the rectal wall after it is exposed in sacrectomy is essential. A prosthetic rectal sling is used in some occasions, but it incurs risk of infection [4]. A free flap is also one of the main options for sacral reconstruction as it can provide sufficient volume to cover the dead space, but it requires an additional donor site and is considered only as a final option. Vertical rectus abdominis myocutaneous (VRAM) flaps could also be considered, but as stated earlier, they have considerable rates of wound complications. As VRAM needs to be transferred transabdominally,

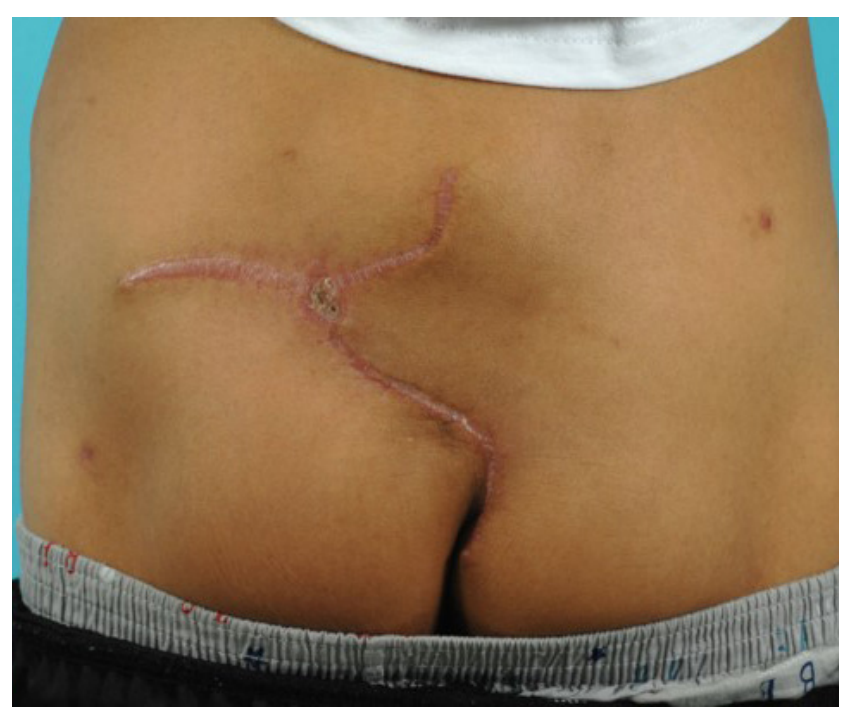

Fig. 3. Postoperative 6 weeks photograph after defect coverage. 

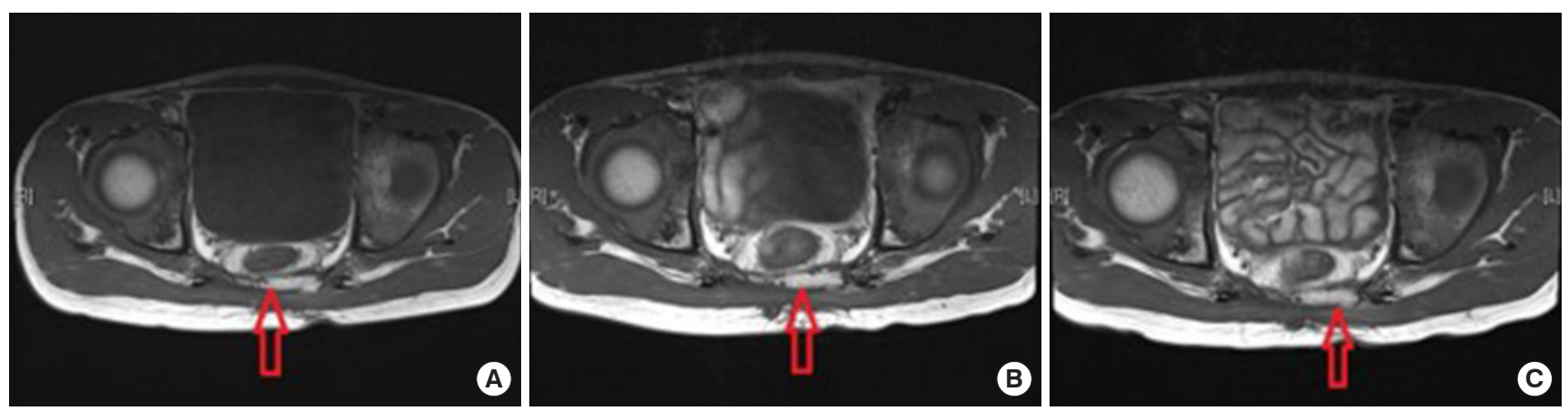

Fig. 4. Magnetic resonance imaging of sacral area. (A) Postoperative 17 months. (B) Postoperative 21 months. (C) Postoperative 25 months. Size of buried superior gluteal perforator flap is maintained. Marked arrow is the buried flap.

flap circulation could be further hindered, leading to high rates of flap necrosis of around 25\% [5].

In this case, as the SGAP penetrates the GM muscle, if this muscle is split once, a flap could be tunneled through the GM muscle to cover the posterior rectal wall. Using an autologous flap lowers the risk of infection relative to that of prosthesis and could also provide sufficient volume to cover the dead space. Furthermore, it is less challenging and easier to perform than a free flap and has no additional donor site morbidity. Atrophy of the flap can be checked using MRI. In conclusion, a buried SGAP flap is a good reconstruction option with minimal morbidity for large post-sacrectomy defects, satisfying both the functional and aesthetic needs of patients.

\section{Conflict of interest}

No potential conflicts of interest relevant to this article are reported.

\section{ORCID iDs}

Seung Heon Hong https://orcid.org/0000-0003-3275-7764

Kyeong-Tae Lee https://orcid.org/0000-0002-9070-9296

\section{References}

1. Maricevich M, Maricevich R, Chim H, et al. Reconstruction following partial and total sacrectomy defects: an analysis of outcomes and complications. J Plast Reconstr Aesthet Surg 2014;67:1257-66.

2. Di Mauro D, D’Hoore A, Penninckx F, et al. V-Y Bilateral gluteus maximus myocutaneous advancement flap in the reconstruction of large perineal defects after resection of pelvic malignancies. Colorectal Dis 2009;11:508-12.

3. Daigeler A, Simidjiiska-Belyaeva M, Drucke D, et al. The versatility of the pedicled vertical rectus abdominis myocutaneous flap in oncologic patients. Langenbecks Arch Surg 2011;396:1271-9.

4. Kim JE, Pang J, Christensen JM, et al. Soft-tissue reconstruction after total en bloc sacrectomy. J Neurosurg Spine 2015;22:571-81.

5. Glatt BS, Disa JJ, Mehrara BJ, et al. Reconstruction of extensive partial or total sacrectomy defects with a transabdominal vertical rectus abdominis myocutaneous flap. Ann Plast Surg 2006;56:526-30. 